\title{
Jatrorrhizine hydrochloride attenuates hyperlipidemia in a high-fat diet-induced obesity mouse model
}

\author{
WEIWEI YANG ${ }^{1 *}$, LIPING SHE ${ }^{2 *}$, KUN YU $^{3}$, SHAN YAN $^{3}$, XUEFENG ZHANG ${ }^{3}$, \\ XIAOYI TIAN ${ }^{3}$, SHUREN MA ${ }^{3}$ and XIWEN ZHANG ${ }^{3}$ \\ ${ }^{1}$ Department of Nephrology, Huai'an First People's Hospital, Nanjing Medical University, Huai'an, Jiangsu 223300; \\ ${ }^{2}$ Department of Intensive Care Unit, Jiangsu Province Geriatric Hospital, Nanjing, Jiangsu 210000; \\ ${ }^{3}$ Department of Cardiology, Huai'an First People's Hospital, Nanjing Medical University, Huai'an, Jiangsu 223300, P.R. China
}

Received October 16, 2015; Accepted July 8, 2016

DOI: $10.3892 / \mathrm{mmr} .2016 .5634$

\begin{abstract}
Jatrorrhizine hydrochloride ( $\mathrm{JH})$ is an active component of the traditional Chinese herb Coptis chinensis, which has been used to prevent and treat metabolic disorders. Hyperlipidemia is one of the principal factors underlying numerous metabolic diseases, including diabetes and obesity. Therefore, the aim of the present study was to investigate the lipid lowering effects of $\mathrm{JH}$ treatment in vivo in an obesity mouse model. JH-treated hyperlipidemic mice exhibited a reduction in body weight, as well as improved glucose tolerance and insulin sensitivity. In addition, JH-treated hyperlipidemic mice exhibited reduced serum triglyceride, total cholesterol and low-density lipoprotein cholesterol levels, as well as increased high-density lipoprotein cholesterol levels compared with untreated mice fed a high-fat diet. Notably, JH treatment ameliorated the pathophysiological changes observed in the livers of hyperlipidemic mice. At the molecular level, JH downregulated the hepatic mRNA expression levels of SREBP-1c and FAS, and induced PPAR- $\alpha$ and CPT1A mRNA expression in hyperlipidemic mice. These findings suggest that $\mathrm{JH}$ ameliorates hyperlipidemia via the suppression of lipogenesis and the enhancement of lipid oxidation in the liver.
\end{abstract}

\section{Introduction}

Hyperlipidemia comprises a heterogeneous group of disorders, characterized by increased levels of one or more lipids and/or lipoproteins in the circulation, including increased

Correspondence to: Dr Xiwen Zhang, Department of Cardiology, Huai'an First People's Hospital, Nanjing Medical University, 6 Beijing West Road, Huai'an, Jiangsu 223300, P.R. China

E-mail: zhangxiwen303@163.com

*Contributed equally

Key words: jatrorrhizine hydrochloride, hyperlipidemia, lipid metabolism, hepatic steatosis, liver levels of atherogenic free fatty acids, triglycerides (TG; known as hypertriglyceridemia), low-density lipoprotein cholesterol (LDL-C; known as hypercholesterolemia) and apolipoprotein B; in addition, decreased levels of antiatherogenic high-density lipoprotein cholesterol (HDL-C) are observed $(1,2)$. The increased prevalence of hyperlipidemia is a public and economic problem worldwide. Hyperlipidemia can arise from the adoption of unhealthy lifestyle choices, such as consuming an excess of high-fat food (3) and alcohol (4), or as a result of specific diseases, including diabetes (5), hypothyroidism (6), erythematosus (7) and chronic renal diseases (8). Hyperlipidemia is a risk factor for cardiovascular disease (CVD), which is the most common cause of mortality worldwide (9). In addition to CVD, hyperlipidemia is closely associated with diabetes, insulin resistance and obesity $(10,11)$. Considering the serious outcomes of this disease, strategies for the prevention and treatment of hyperlipidemia are urgently required and may have a significant clinical value.

Pharmacotherapy is the current primary method for the treatment of hyperlipidemia. Specific agents, including statins (12), fibrates (13), nicotinic acids (14) and bile acid sequestrants (15), have been demonstrated to possess reliable lipid lowering effects and thus dominate the main drug market. However, certain patients are intolerant to these drugs due to their adverse effects, which include liver and kidney toxicity (16), rhabdomyolysis (17) and hyperglycemia (18). This has led to concerns regarding the safety of these drugs for clinical use. Therefore, the search for alternative therapies has gained significant attention in recent years. Traditional Chinese medicine (TCM) products are frequently considered to be less toxic than synthetic agents, and may be a preferred option for the treatment of hyperlipidemia (19). Over the last few decades, hundreds of TCM compounds (20), extracts (21), single herbs (22) or formulae (23) have been reported to be effective in the prevention and treatment of hyperlipidemia, particularly in cases caused by high-fat diet (HFD).

One particular TCM herb, Coptis chinensis (C. chinensis), obtained from the dry rootstocks of $C$. chinensis Franch. or Coptis teeta Wall., has been used to treat gastrointestinal disorders and diarrhea for 30 years worldwide (24). Jatrorrhizine hydrochloride $(\mathrm{JH})$ is an active component of the $C$. chinensis herb and has been demonstrated to have anti-inflammatory (25), 
antimicrobial (26) and leukemia-prevention activities (27). Notably, a recent study revealed that $\mathrm{JH}$ stimulated insulin secretion and decreased fasting blood glucose levels in rats, indicating that this compound may have beneficial effects on energy metabolism (28). Although the protective effects of $\mathrm{JH}$ against hypercholesterolemia and diabetes have been reported by independent studies $(29,30)$, to the best of our knowledge, no comprehensive investigation has been conducted to examine its versatile functions in the regulation of glucose and lipid metabolism. In the present study, an HFD-induced hyperlipidemic mouse model was employed to evaluate the effects of $\mathrm{JH}$ in vivo. $\mathrm{JH}$ was observed to prevent the development of hyperlipidemia in these mice, possibly via the suppression of fatty acid synthesis and by increasing the $\beta$-oxidation of lipids in the liver.

\section{Materials and methods}

Animals. Animal procedures employed in the present study conformed to the Guide for the Care and Use of Laboratory Animals published by the US National Institutes of Health (NIH publication no. 85-23; revised 1996) and the approved regulations established by the Laboratory Animal Care Committee at the Huai'an First People's Hospital Affiliated to Nanjing Medical University (Huai'an, China; permit no. SYXK2016-0016, issued April6, 2016). A total of 28 7-week-old male C57BL/6 mice, weighing 18-22 g, were purchased from the Model Animal Research Center of Nanjing University (Nanjing, China) and maintained under a 12-h light/dark cycle, in a temperature and humidity-controlled environment. Mice were acclimated to lab conditions for 1 week and then divided into four groups ( $\mathrm{n}=7$ mice per group), including the normal diet (ND), the hyperlipidemia model (HFD), HFD and low-dose JH (HFD+JH-L), and HFD and high-dose JH $(\mathrm{HFD}+\mathrm{JH}-\mathrm{H})$ groups. In order to induce hyperlipidemia in the mice, a HFD (60\% fat content; Research Diets, Inc., New Brunswick, NJ, USA) was given to the HFD, HFD+JH-L and HFD+JH-H group mice, as described previously (3); by contrast, ND control group mice were fed a normal rodent chow diet. Mice in the JH-L and JH-H treatment groups received daily intragastric injections of 20 and $100 \mathrm{mg} / \mathrm{kg}$ body weight $\mathrm{JH}$, respectively, while mice in the control and hyperlipidemic groups received an equivalent volume of $0.9 \%$ saline solution using the same procedure. The doses of JH administered to mice were selected based on a previous study demonstrating that $100 \mathrm{mg} / \mathrm{kg} \mathrm{JH}$ decreases blood glucose levels in diabetic mice (28). Mouse body weights were measured every 3 days. Treatment was administered over the course of 8 weeks. Upon completion of treatment, mice were fasted for $6 \mathrm{~h}$ and sacrificed by cervical dislocation for the collection of blood and liver samples. JH (purity, >98\%; Fig. 1) extracted from the tuberous roots of Tinospora sagittata (Oliv.) Gagnep. was purchased from Nanjing Zelang Medical Technology Co., Ltd. (Nanjing, China).

Glucose and insulin tolerance tests. In order to conduct a glucose tolerance test, mice were fasted for $16 \mathrm{~h}$ and then injected intraperitoneally with $1 \mathrm{~g} / \mathrm{kg}$ body weight glucose. To conduct an insulin tolerance test, mice were fasted for $6 \mathrm{~h}$ and then injected intraperitoneally with $0.75 \mathrm{U} / \mathrm{kg}$ body weight insulin. Blood glucose levels were measured prior to injection and at $0,15,30,60$ and 90 min after injection with glucose or insulin using a glucose monitor (Roche Diagnostics, Basel, Switzerland) after injection.

Serological analysis. Blood samples were collected in non-heparinized tubes and centrifuged at $1,000 \mathrm{x} \mathrm{g}$ for 10 min at $4^{\circ} \mathrm{C}$. Kits purchased from the Nanjing Jiancheng Bioengineering Institute (Nanjing, China) were used to determine the levels of serum TG (GPO-PAP assay; catalog no. A110-1), total cholesterol (TC; GPO-PAP assay; catalog no. A111-1), LDL-C (catalog no. A113-1), HDL-C (catalog no. A112-1), aspartate transaminase (AST; catalog no. C010-2) and alanine aminotransferase (ALT; catalog no. C009-2), by spectrophotometric analysis.

Liver histology. Mouse livers were isolated, fixed in a $4 \%$ paraformaldehyde solution for $24 \mathrm{~h}$ in situ, paraffin embedded and cut into $4-\mu \mathrm{m}$ transverse sections for routine hematoxylin-eosin (H\&E) staining.

Reverse transcription-quantitative polymerase chain reaction $(R T-q P C R)$. Total RNA was extracted from mouse livers using the TRIzol reagent (Invitrogen; Thermo Fisher Scientific, Inc., Waltham, MA, USA). Total RNA ( $1 \mu \mathrm{g})$ was then reverse-transcribed into cDNA using PrimeScript ${ }^{\mathrm{TM}}$ RT Master Mix (Takara Bio, Inc., Otsu, Japan) under the following conditions: $37^{\circ} \mathrm{C}$ for $15 \mathrm{~min}$ and then $85^{\circ} \mathrm{C}$ for $5 \mathrm{sec}$. Target gene mRNA levels were determined by qPCR analysis using the SYBR premix Ex Taq (Takara Bio, Inc.) and the LightCycler Nano system (Roche Diagnostics). The primer sequences were as follows: 36B4, sense, 5'-GAAACT GCTGCCTCACATCCG-3', and antisense, 5'-GCTGGCACA GTGACCTCACACG-3'; sterol regulatory element binding transcription factor 1c (SREBP-1c) sense, 5'-GATCAAAGA GGAGCCAGTGC-3', and antisense, 5'-TAGATGGTGGCT GCTGAGTG-3'; fatty acid synthase (FAS) sense, 5'-TCC TGGAACGAGAACACGATCT-3', and antisense, 5'-GAG ACGTGTCACTCCTGGACTTG-3'; peroxisome proliferator activated receptor- $\alpha$ (PPAR $\alpha$ ) sense, 5'-ACGATGCTGTCC TCCTTGATG-3', and antisense, 5'-ACGATGCTGTCCTCC TTGATG-3'; and carnitine palmitoyltransferase 1A (CPT1A) sense, 5'-CTCAGTGGGAGCGACTCTTCA-3', and antisense, 5'-GGCCTCTGTGGTACACGACAA-3'. The qPCR reaction mixture $(10 \mu \mathrm{l})$ consisted of $0.3 \mu \mathrm{l}$ forward primer $(10 \mu \mathrm{M})$, $0.3 \mu 1$ reverse primer $(10 \mu \mathrm{M}), 5 \mu 1$ SYBR Premix Ex Taq (2X), $2 \mu \mathrm{l}$ cDNA template and $2.4 \mu \mathrm{l}$ double-distilled $\mathrm{H}_{2} \mathrm{O}$. Thermal cycling parameters were as follows: $95^{\circ} \mathrm{C}$ for $10 \mathrm{sec}$, followed by 40 cycles of $95^{\circ} \mathrm{C}$ for $5 \mathrm{sec}$ and $60^{\circ} \mathrm{C}$ for $20 \mathrm{sec}$. The 36B 4 gene was used as an internal control. In detail, a quatification cycle (Cq value) was obtained for each amplification curve, and a $\Delta \mathrm{Cq}$ value was first calculated by subtracting the $\mathrm{Cq}$ value for 36B4 from the $\mathrm{Cq}$ value for each sample. Fold-changes compared with the endogenous control were then determined by calculating $2^{-\Delta \Delta \mathrm{Cq}}(31)$.

Western blotting. To determine the protein expression levels of FAS and PPAR $\alpha$, mouse liver samples were first lysed in radioimmunoprecipitation assay buffer, which contained $50 \mathrm{mM}$ Tris/ $\mathrm{HCl}$ (pH 8.0), $150 \mathrm{mM} \mathrm{NaCl}, 1 \%$ Nonidet-P40, 
$1 \%$ sodium deoxycholate, $0.1 \%$ SDS, $0.1 \mathrm{mM}$ DTT, $0.05 \mathrm{mM}$ PMSF, $0.002 \mathrm{mg} / \mathrm{ml}$ aprotinin, $0.002 \mathrm{mg} / \mathrm{ml}$ leupeptin and $1 \mathrm{mM} \mathrm{NaVO}$. Protein concentration was determined using a BCA Protein Quantitation assay kit (catalog no. A045-3; Nanjing Jiancheng Bioengineering Institute). Equal amounts of protein were loaded and separated by $10 \%$ SDS-PAGE prior to transfer onto polyvinylidene fluoride membranes (EMD Millipore, Billerica, MA, USA). Membranes were blocked in $5 \%$ bovine serum albumin blocking buffer ( $\mathrm{pH} 7.5$; Nanjing Sunshine Biotechnology Co., Ltd., Nanjing, China) and incubated with rabbit anti-FAS (1:500; catalog no. 10624-2-AP) and rabbit anti-PPAR $\alpha$ (1:500; catalog no 15540-1-AP) primary polyclonal antibodies (Proteintech Group, Inc., Rosemont, IL, USA), and a mouse anti-GAPDH monoclonal antibody (1:500; catalog no. KC-5G5; KangChen Bio-tech, Inc., Shanghai, China) at $4^{\circ} \mathrm{C}$ overnight. Bound antibodies were detected using the horseradish peroxidase-conjugated secondary antibodies goat anti-rabbit IgG (1:2,000; catalog no. sc-2004) and goat anti-mouse IgG (1:2,000; catalog no. sc-2005) purchased from Santa Cruz Biotechnology, Inc. (Dallas, TX, USA). Membranes were washed with phosphate-buffered saline containing $0.1 \%$ Tween-20 for 10 min between each step. Proteins were visualized using an Enhanced Chemiluminescence kit (catalog no. WBKLS0050; Merck Millipore, Darmstadt, Germany) and quantified using ImageJ software (National Institutes of Health, Bethesda, MA, USA).

Statistical analysis. Data were analyzed using one-way analysis of variance followed by Fisher's least significant difference post-hoc test. Calculations were performed using the Origin8 software (version 8.6; OriginLab, Northampton, MA, USA). Data are presented as the mean \pm standard deviation. $\mathrm{P}<0.05$ was considered to indicate a statistically significant difference.

\section{Results}

Effect of JH on body weight and insulin resistance in HFD-fed mice. As expected, mice consuming an HFD gained substantially more body weight when compared with ND control mice over the course of 8 weeks (Fig. 2A). After 8 weeks of feeding, mice in the HFD group developed severe obesity, as observed by the increase in body weight $(\mathrm{P}<0.001$, HFD vs. ND group; Fig. 2A and B). By contrast, HFD-fed mice treated with JH exhibited a dose-dependent reduction in body weight when compared with untreated HFD-fed mice. Mice in the $\mathrm{JH}-\mathrm{H}$ group exhibited a $27 \%$ decrease in body weight relative to the HFD group after 8 weeks $(\mathrm{P}<0.001, \mathrm{HFD}+\mathrm{JH}-\mathrm{H}$ vs. HFD group). The food intake was reduced in HFD-fed mice compared with ND mice; however, no significant difference in food intake was observed among the HFD-fed groups ( $\mathrm{P}<0.001, \mathrm{HFD}$ vs. ND group; Fig. 2C). Insulin is an important hormone for maintaining blood glucose levels and mediating lipid homeostasis (32). Therefore, serum insulin concentrations in each group were measured. As shown in Fig. 2D, serum insulin concentrations were significantly increased in the HFD model group compared with the ND group. Notably, JH treatment significantly suppressed the HFD-induced increase in insulin concentrations. Greater effects were observed in the HFD+JH-H group $(\mathrm{P}<0.001$, HFD vs. ND group; $\mathrm{P}=0.006$, HFD+JH-L vs. HFD group; $\mathrm{P}<0.001$, HFD+JH-H vs. HFD

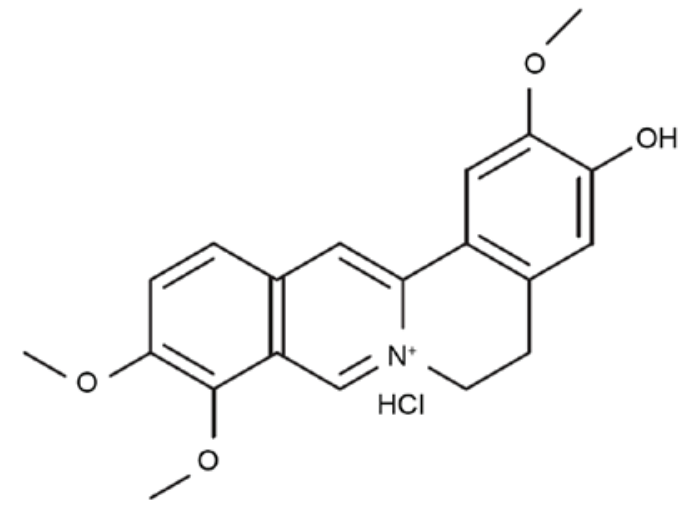

Figure 1. Chemical structure of jatrorrhizine hydrochloride.

group; Fig. 2D). Glucose intolerance and insulin resistance are common syndromes observed in obese individuals (33); since $\mathrm{JH}$ treatment was found to decrease the body weight of obese mice, the effects of $\mathrm{JH}$ treatment on these syndromes was investigated. As shown in Fig. 2E and F, the HFD-fed mice exhibited significantly impaired sensitivities to glucose and insulin compared with the ND-fed mice. Notably, the HFD+JH-H group demonstrated a significantly improved glucose tolerance and insulin sensitivity.

Effect of JH treatment on the serum lipid profile of $H F D$-fed mice. To determine the hypolipidemic effect of JH on HFD-fed mice, the serum levels of TG, TC, LDL-C and HDL-C were measured. As shown in Fig. 3A and B, HFD-fed mice exhibited a significant increase in serum TG and TC levels when compared with the ND group (1.9 and 1.7 -fold increase, $\mathrm{P}<0.001$, HFD vs. ND group). However, $\mathrm{JH}$ treatment significantly reduced the serum TG levels in a dose-dependent manner when compared with the untreated HFD-fed mice, and TG levels in the HFD+JH-H group were comparable to the baseline NC group levels $(\mathrm{P}<0.001$, HFD+JH-L vs. HFD group; $\mathrm{P}<0.001$, HFD+JH-H vs. HFD group; Fig. 3A). Similarly, high-dose JH treatment of HFD-fed mice was associated with a significant reduction in serum TC levels compared with the untreated HFD-fed mice $(\mathrm{P}<0.001$, HFD+JH-H vs. HFD group). However, serum TC levels were reduced to a lesser extent in the $\mathrm{HFD}+\mathrm{JH}-\mathrm{H}$ group $(16 \%$ reduction in the HFD+JH-H vs. HFD group) than the serum TG levels. Furthermore, maintaining a cholesterol balance between potentially harmful LDL-C and beneficial HDL-C is essential for body health (34). As shown in Fig. 3C, JH treatment significantly reduced the HFD-induced upregulation of serum LDL-C levels. By contrast, serum HDL-C levels were significantly elevated following $\mathrm{JH}$ treatment when compared with the untreated HFD-fed mice, with a significant effect observed in the HFD+JH-H group (Fig. 3D). These findings suggest that $\mathrm{JH}$ may ameliorate HFD-induced hyperlipidemia and modify the LDL-C/HDL-C balance by decreasing the ratio between LDL-C and HDL-C.

Effect of JH treatment on hepatic steatosis in HFD-fed mice. As shown in Fig. 4A, morphological changes of the liver, such as enlargement and a yellow color, were observed in the HFD group compared with the ND group. Consistent with these 

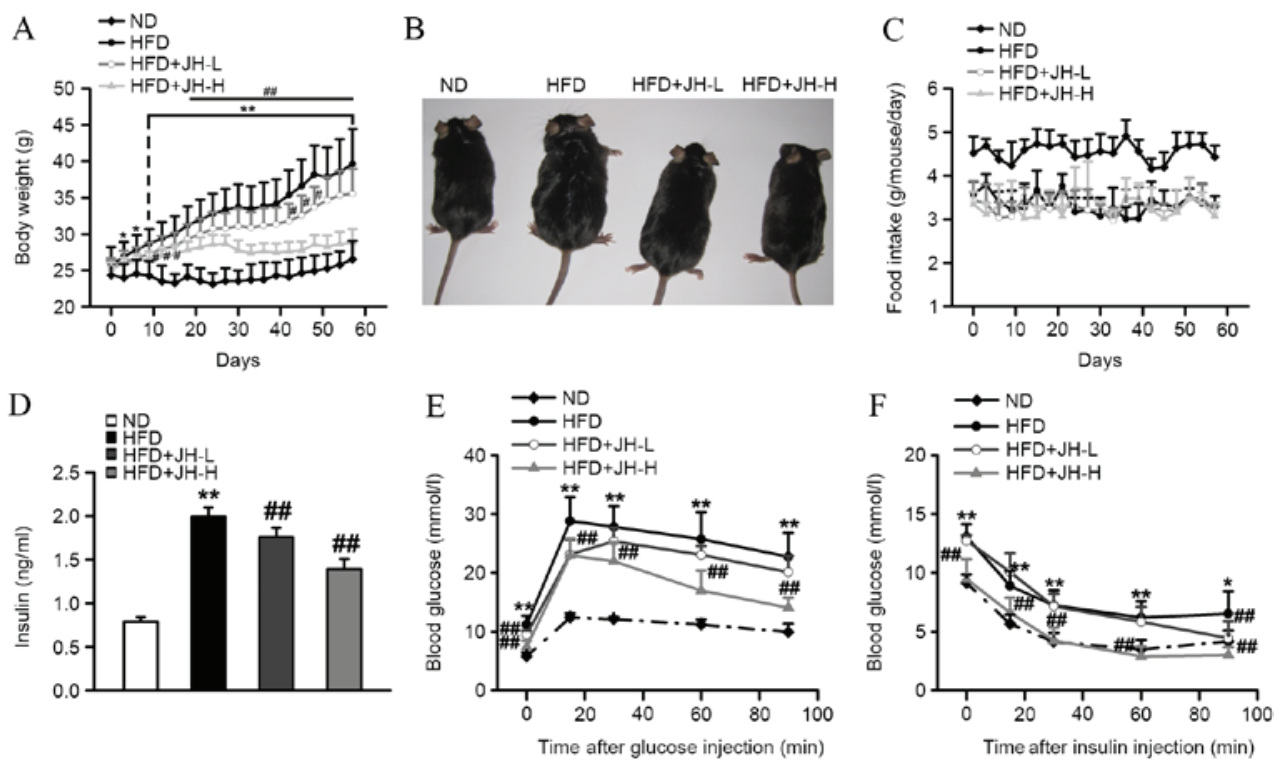

Figure 2. Effect of JH treatment on the body weight, glucose and insulin sensitivity of HFD-fed mice. (A) Body weight, (B) physiology, (C) food intake, (D) serum insulin levels, (E) glucose tolerance test and (F) insulin tolerance test of the ND, HFD, HFD+JH-L (20 mg/kg body weight) and HFD+JH-H $\left(100 \mathrm{mg} / \mathrm{kg}\right.$ body weight) groups are shown ( $\mathrm{n}=7$ per group). Data are presented as the mean \pm standard deviation. ${ }^{*} \mathrm{P}<0.05$ and ${ }^{* *} \mathrm{P}<0.01, \mathrm{HFD}$ vs. ND group; ${ }^{\#} \mathrm{P}<0.05$ and ${ }^{\# \#} \mathrm{P}<0.01, \mathrm{HFD}+\mathrm{JH}-\mathrm{L}$ or HFD+JH-H vs. HFD group. JH, jatrorrhizine hydrochloride; HFD, high-fat diet; ND, normal diet.
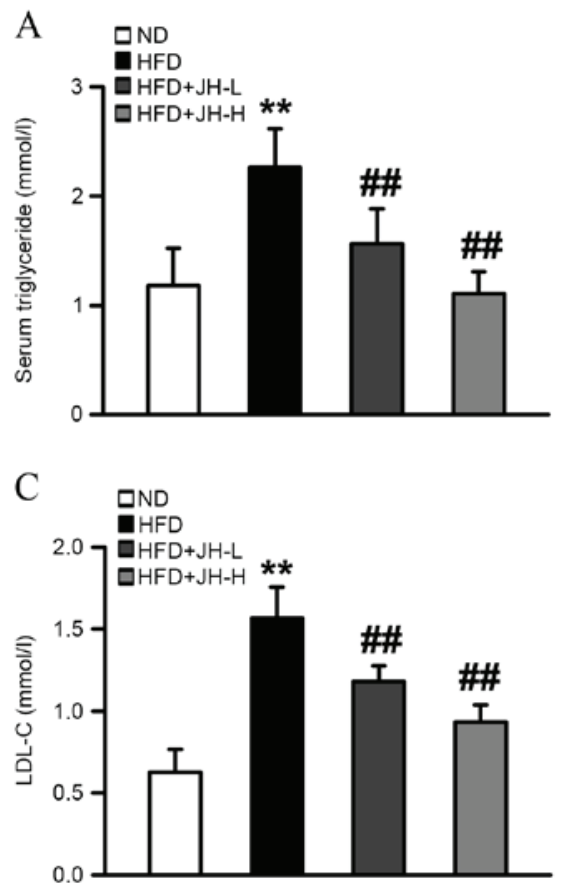
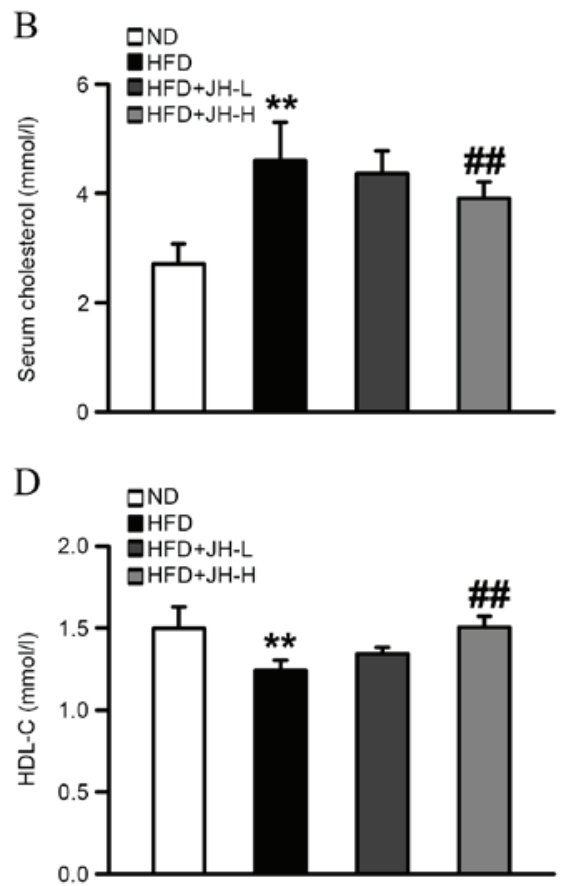

Figure 3. Effect of JH treatment on the serum lipid profile of HFD-fed mice. Serum levels of (A) triglycerides, (B) total cholesterol, (C) LDL-C and (D) HDL-C in the ND, HFD, HFD+JH-L ( $20 \mathrm{mg} / \mathrm{kg}$ body weight) and HFD+JH-H (100 mg $/ \mathrm{kg}$ body weight) groups are shown (n=7 per group). ${ }^{* *} \mathrm{P}<0.01$, HFD vs. ND group; ${ }^{\# /} \mathrm{P}<0.01, \mathrm{HFD}+\mathrm{JH}-\mathrm{L}$ or HFD+JH-H vs. HFD group. Data are presented as the mean \pm standard deviation. JH, jatrorrhizine hydrochloride; HFD, high-fat diet; LDL-C, low-density lipoprotein cholesterol; HDL-C, high-density lipoprotein cholesterol; ND, normal diet.

observations, the liver-to-body weight ratio was significantly increased in the HFD group compared with the ND group due to lipid accumulation in the liver ( $\mathrm{P}=0.001$, HFD vs. ND group; Fig. 4B). By contrast, treatment with JH was shown to reverse these pathological changes and significantly reduce the liver-to-body weight ratio compared with that in untreated HFD-fed mice. Furthermore, H\&E staining was performed to further investigate the effect of JH treatment on HFD-induced lipid accumulation in the liver. As demonstrated in Fig. 4C, lipid droplets were observed in the livers of HFD-fed mice, which was accompanied by the swelling of hepatocytes. However, these pathological alterations were attenuated following treatment with JH. In order to evaluate liver function in all treatment groups, the serum levels of AST and ALT were also determined. As shown in Fig. 4D and E, AST and ALT levels in HFD-fed mice were significantly increased when 
A

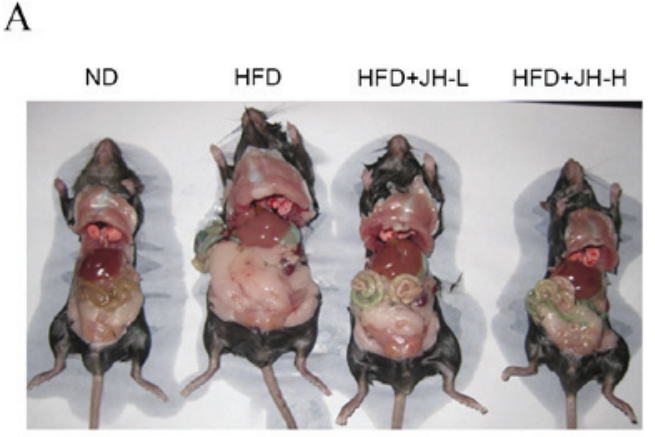

B

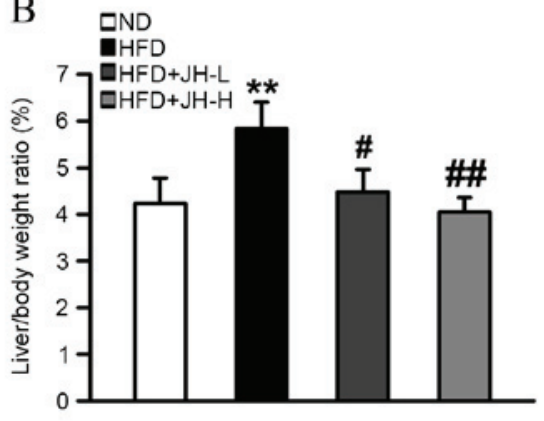

C
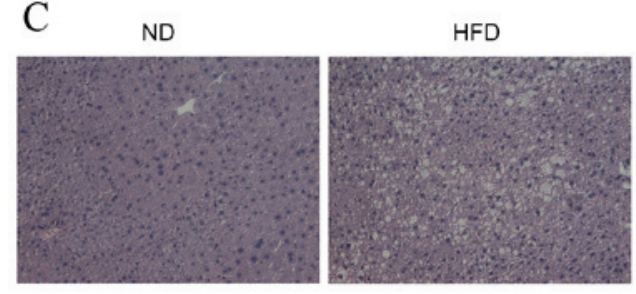

D

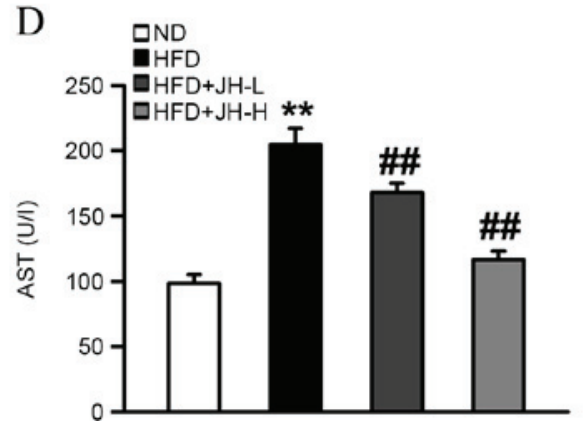

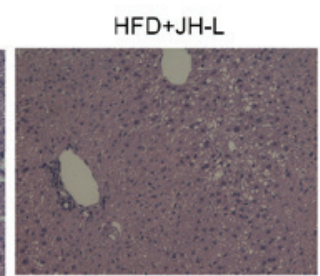

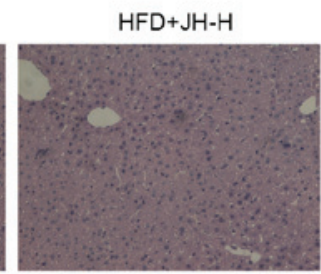

E

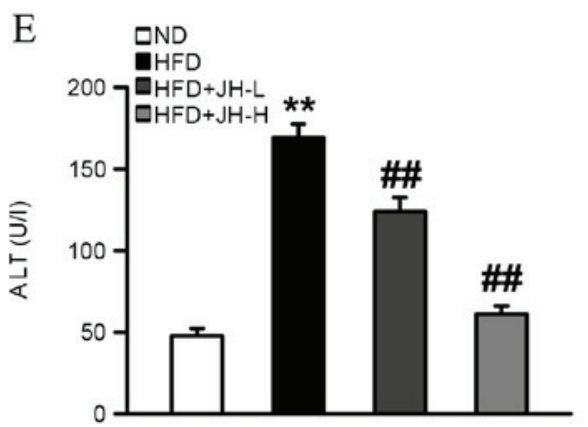

Figure 4. Effect of JH treatment on hepatic steatosis in HFD-fed mice. (A) Liver morphology, (B) liver-to-body weight ratio, (C) liver pathology (as determined by hematoxylin-eosin staining; magnification, x200), (D) serum AST and (E) serum ALT levels in the ND, HFD, HFD+JH-L (20 mg/kg body weight) and HFD+JH-H (100 mg/kg body weight) groups are shown ( $\mathrm{n}=7$ per group). ${ }^{* *} \mathrm{P}<0.01, \mathrm{HFD}$ vs. ND group; ${ }^{*} \mathrm{P}<0.05$ and ${ }^{\# \#} \mathrm{P}<0.01, \mathrm{HFD}+\mathrm{JH}-\mathrm{L}$ or $\mathrm{HFD}+\mathrm{JH}-\mathrm{H}$ vs HFD group. Data are presented as the mean \pm standard deviation. JH, jatrorrhizine hydrochloride; HFD, high-fat diet; AST, aspartate transaminase; ALT, alanine aminotransferase; ND, normal diet.

compared with those in ND-fed mice, which suggests that the liver function was impaired ( $\mathrm{P}<0.001, \mathrm{HFD}$ vs. ND group). However, treatment with $\mathrm{JH}$ resulted in a significant decrease in serum AST and ALT levels when compared to the HFD group ( $\mathrm{P}<0.001, \mathrm{HFD}+\mathrm{JH}-\mathrm{H}$ vs. HFD group; Fig. 4D and E). Therefore, these data preliminarily suggest that JH may counteract the development of obesity and hepatic steatosis.

Effect of JH treatment on the hepatic mRNA levels of lipid-associated genes in HFD-fed mice. Accelerated lipogenesis and decreased fatty acid $\beta$-oxidation occur in the liver during the development of hyperlipidemia (35). To elucidate the molecular mechanisms underlying the hypolipidemic effect of JH, RT-qPCR analysis was employed to quantify the expression levels of hepatic genes involved in the regulation of lipid homeostasis. As shown in Fig. 5A and B, the expression levels of SREBP-1C and FAS, which are lipogenesis-associated genes that promote the synthesis of de novo monounsaturated fatty acids, were significantly increased in the HFD group compared with the ND group $(\mathrm{P}<0.001$, SREBP-1C; $\mathrm{P}=0.026$, FAS). By contrast, the HFD-induced increase in SREBP-1c and FAS expression levels was inhibited by $\mathrm{JH}$ treatment, with a significant effect observed in the HFD+JH-H group ( $\mathrm{P}=0.005$,
SREBP-1C; $\mathrm{P}=0.008$, FAS; HFD+JH-H vs. HFD group). In addition, the hepatic mRNA expression levels of PPAR- $\alpha$ and CPT1A, which are involved in mediating fatty acid $\beta$-oxidation, were significantly decreased in the HFD group compared with the ND group (Fig. 5C and D). However, JH supplementation was associated with a significant increase in PPAR- $\alpha$ and CPT1A mRNA expression levels when compared with the untreated HFD group. Consistent with the mRNA expression levels, the protein expression levels of FAS and PPAR- $\alpha$ were significantly reduced and increased, respectively, following the administration of JH in HFD-fed mice (Fig. 5E). These results suggest that JH may improve HFD-induced hyperlipidemia, in part, through the inhibition of fatty acid synthesis and the activation of fatty acid $\beta$-oxidation in the liver.

\section{Discussion}

The versatile pharmacological activities of $\mathrm{JH}$ have attracted considerable attention in recent years. This compound is a tetrahydroisoquinoline alkaloid and has a similar chemical structure to berberine (36). The reported beneficial effects of $\mathrm{JH}$ include bacteriostasis (37), tumor cell growth suppression (38), reversal of multidrug resistance (39) and the lowering of blood 

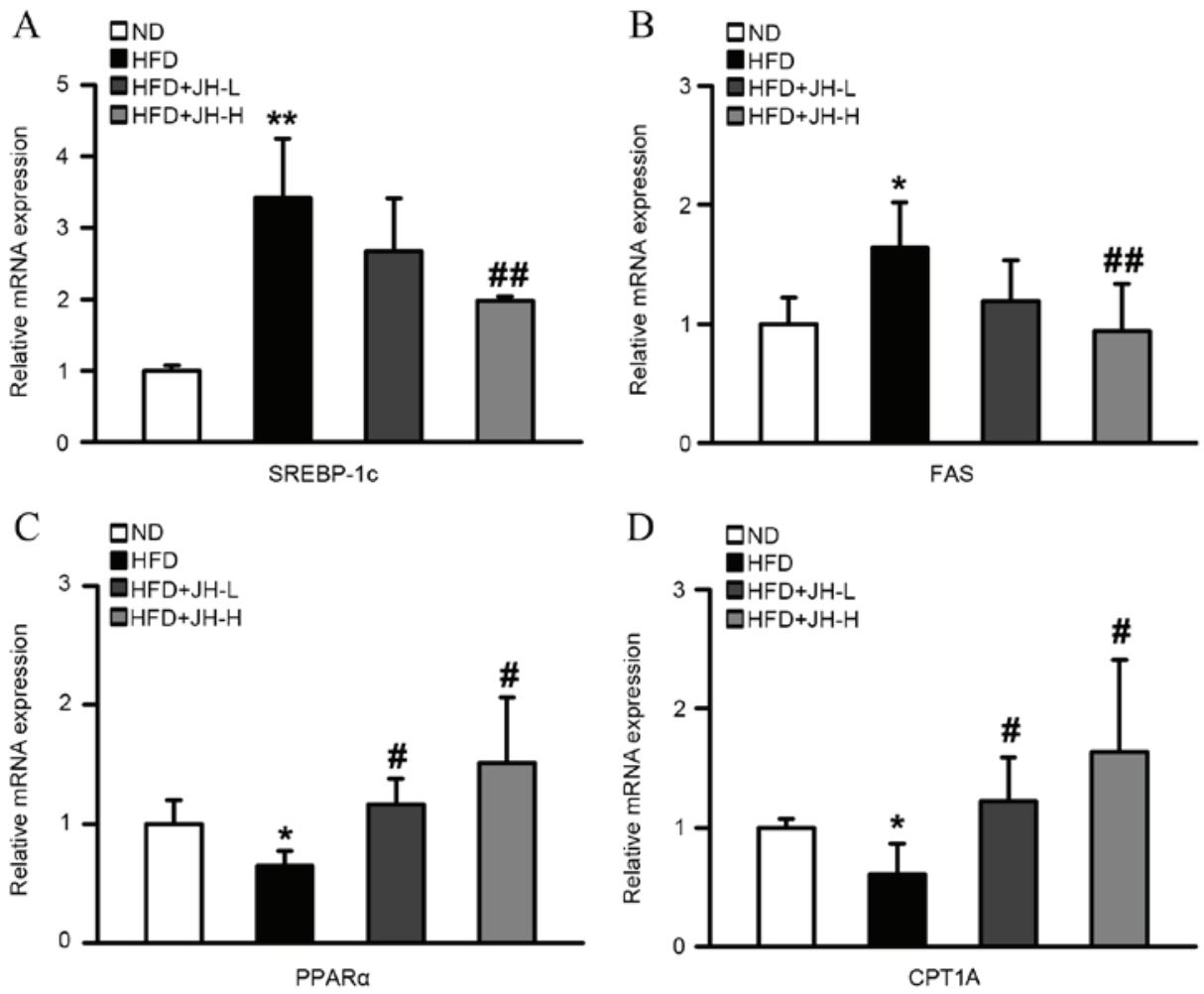

E

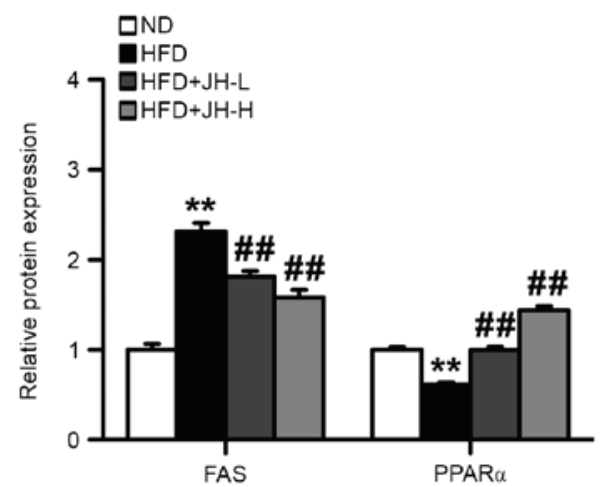

Figure 5. Effect of JH treatment on the hepatic mRNA levels of lipid-associated genes in HFD-fed mice. The mRNA expression levels of (A) SREBP-1c, (B) FAS, (C) PPAR- $\alpha$ and (D) CPT1A in the ND, HFD, HFD+JH-L (20 mg/kg body weight) and HFD+JH-H (100 mg/kg body weight) groups are shown (n=7 per group). (E) Western blot analysis and protein expression levels of FAS and PPAR- $\alpha$ in the various groups are shown. ${ }^{*} \mathrm{P}<0.05$ and ${ }^{* *} \mathrm{P}<0.01, \mathrm{HFD}$ vs. ND group; ${ }^{\#} \mathrm{P}<0.05$ and ${ }^{\# \#} \mathrm{P}<0.01, \mathrm{HFD}+\mathrm{JH}-\mathrm{L}$ or HFD+JH-H vs. HFD group. Data are presented as the mean \pm standard deviation. JH, jatrorrhizine hydrochloride; HFD, high-fat diet; SREBP-1c, sterol regulatory element binding transcription factor 1c; FAS, fatty acid synthase; PPAR- $\alpha$, peroxisome proliferator activated receptor- $\alpha$; CPT1A, carnitine palmitoyltransferase $1 \mathrm{~A} ; \mathrm{ND}$, normal diet.

glucose levels (28). Nevertheless, to the best of our knowledge, no study has investigated the effect of JH on hyperlipidemic animals or humans. The results of the present study suggest that JH may prevent metabolic disorders by ameliorating hyperlipidemia and insulin resistance in HFD-fed obese mice. These beneficial effects may occur via the suppression of lipogenesis and the promotion of fatty acid $\beta$-oxidation.

In the present study, JH inhibited the gain in body weight that was observed in HFD-fed mice in a dose-dependent manner. This effect was not associated with food intake, as no notable differences in diet consumption were observed among the HFD-fed groups, suggesting that JH treatment may not affect appetite. Obesity is known to be the most common cause of hepatic steatosis, which is characterized by the pathological accumulation of fat in the liver, and leads to liver damage in the form of inflammation and fibrosis (40). In the current study, hepatic steatosis was observed in HFD-fed mice, which was evidenced by alterations in liver morphology (enlargement and a yellow color) and histology (increased lipid droplet accumulation). Notably, these pathological changes were reversed by $\mathrm{JH}$ treatment. In addition, serum AST and ALT levels, a hallmark of liver injury (41), were significantly downregulated by $\mathrm{JH}$ treatment in HFD-fed mice. Therefore, JH may be useful for the treatment of fatty liver disease caused by hyperlipidemia. In addition, the severe glucose intolerance, insulin resistance and hyperinsulinemia observed in HFD-fed mice was significantly attenuated by JH treatment. Taken together with its hypoglycemic effects, JH may be a potential therapeutic agent for the prevention of diabetes. However, it should be noted that the present study was designed for investigating 
$\mathrm{JH}$ as a preventative agent for metabolic disorders. JH was administered to mice when hyperlipidemia had not been fully induced. Future studies should assess the protective roles of $\mathrm{JH}$ in a therapeutic setting, with an extension of treatment time.

Lipid homeostasis is dependent on the balance between lipogenesis (storage) and fatty acid $\beta$-oxidation (consumption). A previous study demonstrated that the SREBP-1c and PPAR $\alpha$ transcription factors and the expression of their respective target genes, FAS and CPT1A, serve crucial roles in the development of hyperlipidemia (42). SREBP-1c modulates the expression of a large number of genes involved in the uptake of lipoproteins, and the synthesis of cholesterol, TG and very-low-density lipoprotein cholesterol in the liver (43). In addition, PPAR $\alpha$ regulates the expression of genes involved in mitochondrial and liver fatty acid $\beta$-oxidation $(44,45)$. In the present study, HFD increased the hepatic mRNA expression levels of SREBP1c and FAS, but decreased the levels of hepatic PPAR- $\alpha$ and CPT1A. Notably, treatment of HFD-fed mice with $\mathrm{JH}$ reversed these effects. These data suggest that reducing the expression levels of genes that inhibit lipogenesis (namely SREBP-1c and FAS) and upregulating the expression levels of genes involved in promoting lipid metabolism (namely PPAR $\alpha$ and CPT1A) may underlie the beneficial effects of $\mathrm{JH}$ on HFD-induced hyperlipidemia and liver damage in mice.

FAS and CPT1A are important enzymes involved in hepatic lipogenesis and fatty acid $\beta$-oxidation. FAS catalyzes the final step of the fatty acid biosynthesis process, while CPT1A facilitates the transfer of long-chain fatty acids across the mitochondrial membrane during $\beta$-oxidation (46). In the present study, the mRNA expression levels of genes encoding these enzymes were analyzed. JH treatment of HFD-fed mice suppressed FAS and promoted CPT1A mRNA expression levels. It should be noted that these results provide a preliminary insight into the mechanisms through which $\mathrm{JH}$ exerts its pharmacological functions. Future studies should conduct additional experiments in order to elucidate the beneficial functions of $\mathrm{JH}$ in the prevention and/or treatment of metabolic disorders. For instance, the protein expression and enzymatic activity levels of these enzymes should be determined. In addition, gain and loss-of-function strategies could be employed to artificially manipulate FAS and CPT1A expression, in order to determine whether these enzymes mediate the effects of $\mathrm{JH}$ on hyperlipidemia.

In conclusion, the results of the present study suggest that JH may serve a role in ameliorating metabolic disorders in HFD-fed obese mice. The underlying mechanisms of the hypolipidemic effect of $\mathrm{JH}$ may involve the suppression of lipogenesis and the promotion of fatty acid $\beta$-oxidation. These results provide novel insights into the role of $\mathrm{JH}$ in regulating liver energy metabolism, and suggest that treatment with $\mathrm{JH}$ may present an effective and safe strategy for the prevention of hyperlipidemia and other metabolic disorders. Further investigation is required to elucidate the mechanisms by which this compound protects against metabolic disorders and to determine whether additional mechanisms are involved.

\section{Acknowledgements}

This study was supported by grants from the Applied and Technologic Research Program of Huai'an (no. HAS2014009) and the Research Fund for the Technology Development Project of Nanjing Medical University (no. 2013NJMU226).

\section{References}

1. Betteridge DJ: Diabetic dyslipidaemia. Eur J Clin Invest 29 (Suppl 2): S12-S16, 1999.

2. Goldberg IJ: Clinical review 124: Diabetic dyslipidemia: Causes and consequences. J Clin Endocrinol Metab 86: 965-971, 2001.

3. Lin J, Yang R, Tarr PT, Wu PH, Handschin C, Li S, Yang W, Pei L, Uldry M, Tontonoz P, et al: Hyperlipidemic effects of dietary saturated fats mediated through PGC-1beta coactivation of SREBP. Cell 120: 261-273, 2005.

4. Mendelson JH and Mello NK: Alcohol-induced hyperlipidemia and beta lipoproteins. Science 180: 1372-1374, 1973.

5. Dunn FL: Hyperlipidemia in diabetes mellitus. Diabetes Metab Rev 6: 47-61, 1990.

6. Karthick N, Dillara K, Poornima KN and Subhasini AS: Dyslipidaemic changes in women with subclinical hypothyroidism. J Clin Diagn Res 7: 2122-2125, 2013.

7. Borba EF and Bonfá E: Dyslipoproteinemias in systemic lupus erythematosus: Influence of disease, activity, and anticardiolipin antibodies. Lupus 6: 533-539, 1997.

8. Sentí M, Romero R, Pedro-Botet J, Pelegrí A, Nogués X and Rubiés-Prat J: Lipoprotein abnormalities in hyperlipidemic and normolipidemic men on hemodialysis with chronic renal failure. Kidney Int 41: 1394-1399, 1992.

9. O'Keefe JH and Bell DS: Postprandial hyperglycemia/hyperlipidemia (postprandial dysmetabolism) is a cardiovascular risk factor. Am J Cardiol 100: 899-904, 2007.

10. Haffner SM: Diabetes, hyperlipidemia, and coronary artery disease. Am J Cardiol 83: 17F-21F, 1999.

11. O'Brien T, Nguyen TT and Zimmerman BR: Hyperlipidemia and diabetes mellitus. Mayo Clin Proc 73: 969-976, 1998.

12. Farnier M and Davignon J: Current and future treatment of hyperlipidemia: The role of statins. Am J Cardiol 82: 3J-10J, 1998.

13. Staels B, Dallongeville J, Auwerx J, Schoonjans K, Leitersdorf E and Fruchart JC: Mechanism of action of fibrates on lipid and lipoprotein metabolism. Circulation 98: 2088-2093, 1998.

14. Garg A and Grundy SM: Nicotinic acid as therapy for dyslipidemia in non-insulin-dependent diabetes mellitus. JAMA 264: 723-726, 1990.

15. Ast $\mathrm{M}$ and Frishman WH: Bile acid sequestrants. J Clin Pharmacol 30: 99-106, 1990.

16. Akoglu H, Yilmaz R, Kirkpantur A, Arici M, Altun B and Turgan C: Combined organ failure with combination antihyperlipidemic treatment: A case of hepatic injury and acute renal failure. Ann Pharmacother 41: 143-147, 2007.

17. Thompson PD, Clarkson P and Karas RH: Statin-associated myopathy. JAMA 289: 1681-1690, 2003.

18. Miettinen TA, Taskinen MR, Pelkonen R and Nikkilä EA: Glucose tolerance and plasma insulin in man during acute and chronic administration of nicotinic acid. Acta Med Scand 186: 247-253, 1969.

19. Ho YP, To KK, Au-Yeung SC, Wang X, Lin G and Han X: Potential new antitumor agents from an innovative combination of demethylcantharidin, a modified traditional Chinese medicine, with a platinum moiety. J Med Chem 44: 2065-2068, 2001.

20. Hsu CL, Wu CH, Huang SL and Yen GC: Phenolic compounds rutin and o-coumaric acid ameliorate obesity induced by high-fat diet in rats. J Agric Food Chem 57: 425-431, 2009.

21. Park SH, Ko SK, Choi JG and Chung SH: Salicornia herbacea prevents high fat diet-induced hyperglycemia and hyperlipidemia in ICR mice. Arch Pharm Res 29: 256-264, 2006.

22. Chang XX, Yan HM, Xu Q, Xia MF, Bian H, Zhu TF and Gao X: The effects of berberine on hyperhomocysteinemia and hyperlipidemia in rats fed with a long-term high-fat diet. Lipids Health Dis 11: 86, 2012.

23. Feng Q, Gou XJ, Meng SX, Huang C, Zhang YQ, Tang YJ, Wang WJ, Xu L, Peng JH and Hu YY: Qushi huayu decoction inhibits hepatic lipid accumulation by activating AMP-Activated protein kinase in vivo and in vitro. Evid Based Complement Alternat Med 2013: 184358, 2013.

24. Khin-Maung-U, Myo-Khin, Nyunt-Nyunt-Wai, Aye-Kyaw and Tin-U: Clinical trial of berberine in acute watery diarrhoea. $\mathrm{Br}$ Med J (Clin Res Ed) 291: 1601-1605, 1985. 
25. Akhter MH, Sabir M and Bhide NK: Anti-inflammatory effect of berberine in rats injected locally with cholera toxin. Indian J Med Res 65: 133-141, 1977.

26. Amin AH, Subbaiah TV and Abbasi KM: Berberine sulfate: Antimicrobial activity, bioassay, and mode of action. Can J Microbiol 15: 1067-1076, 1969.

27. Lin CC, Ng LT, Hsu FF, Shieh DE and Chiang LC: Cytotoxic effects of Coptis chinensis and Epimedium sagittatum extracts and their major constituents (berberine, coptisine and icariin) on hepatoma and leukaemia cell growth. Clin Exp Pharmacol Physiol 31: 65-69, 2004.

28. Fu Y, Hu B, Tang Q, Fu Q, Zhang Q and Xiang JZ: Effect of jatrorrhizine, berberine, Huanglian Decoction and compound-mimic prescription on blood glucose in mice. Chung Tsao Yao Tsa Chih Pien Chi Pu 36: 548-551, 2005 (In Chinese).

29. Wu H,He K, Wang Y, Xue D, Ning N, Zou Z, Ye X, Li X, Wang D and Pang J: The antihypercholesterolemic effect of jatrorrhizine isolated from Rhizoma Coptidis. Phytomedicine 21: 1373-1381, 2014.

30. Fu Y, Hu B, Tang Q, Fu Q and Xiang J: Hypoglycemic activity of jatrorrhizine. J Huazhong Univ Sci Technolog Med Sci 25: 491-493, 2005.

31. Livak KJ and Schmittgen TD: Analysis of relative gene expression data using real-time quantitative PCR and the 2(-Delta Delta C(T)) Method. Methods 25: 402-408, 2001

32. Saltiel AR and Kahn CR: Insulin signalling and the regulation of glucose and lipid metabolism. Nature 414: 799-806, 2001.

33. Kopelman PG: Obesity as a medical problem. Nature 404: 635-643, 2000.

34. Toth PP: Cardiology patient page. The 'good cholesterol': High-density lipoprotein. Circulation 111: e89-e91, 2005.

35. Wiegman $\mathrm{CH}$, Bandsma RH, Ouwens M, van der Sluijs FH, Havinga R, Boer T, Reijngoud DJ, Romijn JA and Kuipers F: Hepatic VLDL production in ob/ob mice is not stimulated by massive de novo lipogenesis but is less sensitive to the suppressive effects of insulin. Diabetes 52: 1081-1089, 2003.
36. Liu F, Li Z, Shi X and Zhong M: Determination of berberine, palmatine and jatrorrhizine in rabbit plasma by liquid chromatography-electrospray ionization-mass spectrometry. J Pharm Biomed Anal 56: 1006-1015, 2011.

37. Kong W, Zhao Y, Xiao X, Jin C, Liu Y and Li Z: Comparison of anti-bacterial activity of four kinds of alkaloids in Rhizoma Coptidis based on microcalorimetry.Zhongguohuaxue (yingwenban) 27: 1186-1190, 2009 (In Chinese)

38. Liu R, Cao Z, Pan Y, Zhang G, Yang P, Guo P and Zhou Q: Jatrorrhizine hydrochloride inhibits the proliferation and neovascularization of C8161 metastatic melanoma cells. Anticancer Drugs 24: 667-676, 2013.

39. Xiong $C$ and Fang D: Effects of jatrorrhizine on isolated guinea pig atria. Zhongguo Yaolixue Yu Dulixue Zazhi 4: 255-259, 1989 (In Chinese).

40. Reddy JK and Rao MS: Lipid metabolism and liver inflammation. II. Fatty liver disease and fatty acid oxidation. Am J Physiol Gastrointest Liver Physiol 290: G852-G858, 2006.

41. Renner EL and Dällenbach A: Increased liver enzymes: What should be done? Ther Umsch 49: 281-286, 1992 (In German).

42. Nguyen P, Leray V, Diez M, Serisier S, Le Bloc'h J, Siliart B and Dumon H: Liver lipid metabolism. J Anim Physiol Anim Nutr (Berl) 92: 272-283, 2008.

43. Eberlé D, Hegarty B, Bossard P, Ferré P and Foufelle F: SREBP transcription factors: Master regulators of lipid homeostasis. Biochimie 86: 839-848, 2004.

44. Janani $C$ and Ranjitha Kumari BD: PPAR gamma gene-A review. Diabetes Metab Syndr 9: 46-50, 2015.

45. Pawlak M, Lefebvre P and Staels B: Molecular mechanism of PPAR $\alpha$ action and its impact on lipid metabolism, inflammation and fibrosis in non-alcoholic fatty liver disease. J Hepatol 62: 720-733, 2015.

46. Wakil SJ and Abu-Elheiga LA: Fatty acid metabolism: Target for metabolic syndrome. J Lipid Res 50 (Suppl): S138-S143, 2009. 